\title{
Preliminary quantitative myocardial perfusion in response to cold pressor test in normals
}

\author{
Yi Wang ${ }^{*}$, Mike Passic \\ From 16th Annual SCMR Scientific Sessions \\ San Francisco, CA, USA. 31 January - 3 February 2013
}

\section{Background}

Echo and nuclear studies have shown that coronary flow changes with the cold pressor test (CPT). CPT has been used to measure coronary flow response because it provides an endothelium-dependent coronary vasodilation that is sensitive for detecting early changes in coronary endothelial function. There was also a study showed myocardial perfusion increases during CPT with first pass perfusion imaging [1]. We studied the regional myocardial perfusion values in normal volunteers under stress $(\mathrm{CPT})$, at rest, and myocardial flow reserve (MFR).

\section{Methods}

To ensure the normality of the data, strict exclusion criteria were used in volunteer recruitment, including exclusion of hypertension, diabetes, smoking, family history of cardiac disease, a cardiac ultrasound and CT coronary calcium score $\leq 20$. Three volunteers (ages: $38.3 \pm 9.8,1$ female) were enrolled after IRB approval. All subjects were injected with a $0.05 \mathrm{mM} / \mathrm{kg} \mathrm{Gd}$ at $6 \mathrm{ml} / \mathrm{s}$, with additional $0.005 \mathrm{mM} / \mathrm{kg}$ Gd injection immediately prior to it (dual bolus injection). During the contrast administration, the first pass perfusion imaging under CPT stress was performed on a $1.5 \mathrm{~T}$ scanner (Siemens, Malvern, PA) by putting the right hand in an icy water bath for 90 seconds before the perfusion imaging. A saturation recovery SSFP technique was used for both CPT and the 30-minute later resting perfusion. A voxel spatial resolution of $1.9 \times 2.8 \times 8$ $\mathrm{mm}^{3}$ was achieved in 3 rotational long axis slices per heartbeat over 50 heartbeats. Mean signal intensities of all pixels in each of the six myocardial segment at every time point were transferred to custom developed program to calculate absolute regional myocardial flow. To solve the deconvolution equation, a Fermi function was selected as the distribution of tracer residence times to search for the best fit of the myocardial dynamic signal curve for each sector, with LV blood signal as arterial input function. A total of 18 segments per volunteer were evaluated. The MFR was determined as the ratio of stress to rest perfusion.

\section{Results}

All volunteers were able to keep the hand in the icewater bath for the whole CPT. The mean and standard deviation of rest perfusion for all volunteers were $0.55 \pm$ $0.27 \mathrm{ml} / \mathrm{g} / \mathrm{min}, 0.57 \pm 0.18 \mathrm{ml} / \mathrm{g} / \mathrm{min}$ during CPT, and $1.15 \pm 0.23$ of MFR, respectively, $\mathrm{n}=54$. The mean perfusion between CTP and rest was not significant.

\section{Conclusions}

Absolute quantification of myocardial blood flow with dual bolus injection during CPT is feasible. Contrary to previous study [1], mean perfusion during CPT showed a moderate increase compare to rest, but not significant in Normals. Further studies with Normals and patients with known cardiac risk factors are needed to validate these findings.

\section{Funding}

St. Francis Research Foundation.

Published: 30 January 2013

Reference

1. Weng A, Ritter CO, Kowalski M, Beer MJ, Hahn D, Köstler H: Cold pressor test in MRI for quantitative myocardial perfusion imaging. ISMRM Proceedings 2009.

doi:10.1186/1532-429X-15-S1-E49

Cite this article as: Wang and Passic: Preliminary quantitative

myocardial perfusion in response to cold pressor test in normals. Journal of Cardiovascular Magnetic Resonance 2013 15(Suppl 1):E49.

St. Francis Hospital, Roslyn, NY, USA

\section{() Biomed Central}

(c) 2013 Wang and Passic; licensee BioMed Central Ltd. This is an Open Access article distributed under the terms of the Creative Commons Attribution License (http://creativecommons.org/licenses/by/2.0), which permits unrestricted use, distribution, and reproduction in any medium, provided the original work is properly cited. 